Mots. Les langages du politique

\title{
« La polémique est un mode de gestion socio- discursif du conflit dans les sociétés démocratiques »
}

Entretien avec Ruth Amossy, professeur émérite à l'Université de Tel Aviv, 30 avril 2014. Réalisé et transcrit par Claire Oger

\section{Claire Oger}

\section{OpenEdition}

\section{Journals}

Édition électronique

URL : https://journals.openedition.org/mots/22067

DOI : $10.4000 /$ mots.22067

ISSN : 1960-6001

Éditeur

ENS Éditions

Édition imprimée

Date de publication : 6 octobre 2015

Pagination : 155-165

ISBN : 978-2-84788-727-3

ISSN : 0243-6450

\section{Référence électronique}

Claire Oger, « «La polémique est un mode de gestion socio-discursif du conflit dans les sociétés démocratiques » », Mots. Les langages du politique [En ligne], 108 | 2015, mis en ligne le 06 octobre 2017, consulté le 23 avril 2022. URL : http://journals.openedition.org/mots/22067 ; DOI : https:// doi.org/10.4000/mots.22067 


\section{« La polémique est un mode de gestion socio-discursif du conflit dans les sociétés démocratiques "}

Mots. Les langages du politique : Vous avez publié en 2014 un ouvrage intitulé Apologie de la polémique, un titre doublement inhabituel qui constitue, d'une part, une prise de position et qui valorise, d'autre part, un type de discours généralement réprouvé. Quel sens faut-il donner ici à «apologie»?

Ruth Amossy : L'apologie, c'est d'abord une plaidoirie, une «défense de », si on revient à son sens antique, et en même temps, c'est devenu dans le langage courant l'équivalent de «éloge de». J'ai choisi ce titre - sur lequel j'ai d'ailleurs hésité - précisément parce que la polémique était vilipendée sur la place publique, chacun accusant l'autre de polémiquer et se défendant bien de le faire lui-même. Or quand on regarde ce qui se passe concrètement, on s'aperçoit qu'il y a un nombre extrêmement important de polémiques et que les médias ne cessent de titrer sur elles. Je me retrouvais donc devant un paradoxe, d'une part un phénomène que tout le monde s'accorde à critiquer et à blâmer, et d'autre part une pratique incessante. Il m'est apparu indispensable d'analyser des cas de figure, de façon concrète, sur des corpus précis. En cours de route, j'ai découvert que la polémique remplissait des fonctions importantes qui expliquaient sa pérennité et sa vitalité - ce dont l'ouvrage entend témoigner.

Mots. Les langages du politique : Quels sont les corpus que vous avez choisi d'étudier pour mettre en évidence ces fonctions?

Ruth Amossy : Je me suis concentrée, puisque le livre était en français et destiné principalement à un public français, sur des polémiques publiques françaises, tout en ajoutant un cas relatif à Israël, où je vis et où les polémiques abondent.

Certains passages du livre évoquent de façon rapide des cas particuliers, comme la polémique sur la photo qui avait gagné un prix de la Fnac et qui présentait un individu s'essuyant le postérieur avec un drapeau français, ou encore celle qui a entouré les prises de position de Gérard Depardieu au moment de son exil fiscal. Je me suis cependant attardée davantage sur deux ensembles de polémiques. D’abord, celles relatives au statut des femmes, en France et en Israël : la polémique qui s'est déchaînée autour de la burqa, et qui faisait suite à celles, plus anciennes, sur le voile et dans un deuxième temps, une polémique qui avait 
opposé, en Israël, les ultra-orthodoxes aux autres secteurs de la population (laïcs ou religieux modérés). Elle a éclaté à partir d'un incident mineur mais, comme c'est généralement le cas, révélateur de divisions, de conflits profonds dans la société. Il s'agissait d'une femme qui était montée dans un bus ultra-orthodoxe et qu'on avait priée de s'asseoir à l'arrière, comme c'est la coutume chez eux. Elle a refusé, ce qui a déclenché des réactions très vives chez les ultra-orthodoxes, et le déploiement d'une polémique généralisée dans l'espace public.

Le deuxième corpus est pris dans un domaine tout à fait différent, et concerne les bonus et les stock-options que se distribuaient certains dirigeants, après la grande crise de 2008 , en 2009 : non pas les controverses savantes entre économistes, puisqu'évidemment il y avait des théories fort différentes sur le sujet, mais bien la parole des simples citoyens qui ont désiré s'exprimer, en voyant en particulier comment ils le faisaient sur l'Internet, c'est-à-dire dans les forums de discussion des grands quotidiens, Libération, Le Monde, Le Figaro...

Cela m'a permis d'aborder la polémique telle qu'elle se déroulait dans les médias, qu'il s'agisse d'un forum de discussion ou encore d'un débat télévisé, et de voir comment elle fonctionnait, dans l'espace public, à travers plusieurs dispositifs.

Mots. Les langages du politique : Vous distinguez à ce sujet dans l'ouvrage le fonctionnement de la polémique et ses fonctions. Pouvez-vous en présenter les grandes lignes?

Ruth Amossy : Il faut dans un premier temps définir ce qu'est la polémique : ce n'est pas simplement un débat vif, ou encore violent. Pour qu'il y ait polémique, il faut certes qu'il y ait un antagonisme marqué, un choc de thèses contradictoires, mais cela ne suffit pas. Dans la polémique, les contradictions sont exacerbées et entraînent un phénomène de dichotomisation - c'est ce qu'avait bien mis en valeur Marcelo Dascal ${ }^{1}$ quand il avait étudié le phénomène. Cette dichotomisation consiste à opposer deux réponses divergentes à une question en exacerbant les différences entre les thèses : on hyberbolise ce qui les sépare, il n'y a pas de nuances, c'est blanc ou noir...

Le deuxième trait, c'est la polarisation, qu'il ne faut pas confondre avec la dichotomisation. Cette dernière est un phénomène discursif, tandis que la polarisation est un phénomène social : à partir de l'opposition forte entre les thèses, on regroupe les gens en deux camps ennemis, regroupement qui recouvre des enjeux identitaires forts.

Enfin, il faut signaler le fait que dans la polémique, l'autre se trouve attaqué, à la fois dans sa thèse et dans sa personne : comme Catherine KerbratOrecchioni (1980) l'avait montré, la polémique suppose un discrédit jeté sur l'autre, et le recours fréquent à l'argument ad hominem.

1. Voir par exemple Dascal, 2008. 
Ce sont les traits définitoires de la polémique, auxquels peuvent s'ajouter le cas échéant la passion et la violence, qui sont fréquentes mais pas obligées (et qu'on prend souvent à tort comme constitutives de la polémique).

Il faut souligner que la polémique n'est pas une parole sauvage, c'est une modalité de l'argumentation. En position centrale de l'argumentation se situe le débat rationnel entre deux personnes qui présentent des thèses opposées, principalement sur le mode du logos. Mais il y a d'autres modalités et on peut se représenter le phénomène comme scalaire : à un des pôles, un type d'argumentation qui minimise la confrontation, et peut même ne pas mentionner la thèse de l'autre, effaçant la dimension conflictuelle; à l'autre pôle, une position qui exacerbe la confrontation. Entre ces deux pôles, bien sûr, on trouve de nombreux cas intermédiaires, comme la négociation, la coconstruction des réponses...

Il y a donc plusieurs modalités de l'argumentation et la polémique constitue l'une d'entre elles, que l'on peut étudier dans ses spécificités. Quand on entre dans le détail de la trame discursive, on peut retrouver les différents procédés qui sont mis en œuvre, et la façon dont chacun mobilise tout un arsenal de moyens discursifs et rhétoriques pour mener à bien cette sorte de duel, puisqu'on sait que polémique renvoie au mot grec polemos, c'est une guerre de plume.

Mots. Les langages du politique: Vous avez évoqué les enjeux identitaires de la constitution des groupes sociaux qui s'affrontent dans une polémique. En fait, les deux camps opposés rassemblent des acteurs très différents, ils peuvent être très hétérogènes selon vous du point de vue sociologique, et même idéologique. Quelle est l'importance de ce phénomène?

Ruth Amossy : Oui, c'est un phénomène très intéressant : le but de celui qui propose une thèse et de celui qui s'y oppose, c'est d'amener le plus grand nombre de personnes à sa propre position. Dans cette dynamique de l'argumentation et à travers ce phénomène de polarisation, on pense souvent avoir affaire à des groupes existants, constitués d'avance et qui correspondent aux grands conflits sociaux - et c'est certainement parfois le cas. Mais on observe que souvent, dans une circonstance donnée, des gens extrêmement différents se regroupent autour d'une même position et qu'ils le font à partir de points de vue différents, de croyances différentes, qu'ils se rassemblent en quelque sorte à cette occasion, en s'identifiant au Proposant ou à l'Opposant.

On touche ici à la première fonction de la polémique qui est précisément d'opérer des regroupements dans l'espace social : cela a été le cas en France autour de la question du port du voile. Les polémiques opposent des camps qui ne sont pas historiquement ou sociologiquement constitués, qui ne reflètent pas des rapports de forces ou des positionnements préexistants. L'existence de ces camps opposés résulte au contraire de l'activité polémique elle-même. Et 
c'est d'ailleurs parce que la polémique est une activité discursive qui crée des regroupements et des rapprochements identitaires qu'on voit des politiques essayer de mettre à profit ce phénomène, et s'appuyer sur ces regroupements - en quelque sorte inespérés - pour retrouver une cohérence perdue, pour tenter de récupérer à leur profit ces effets de la polémique.

Mais une autre fonction peut être évoquée, en se plaçant dans un contexte plus large : c'est la mise en contact des deux camps, qui permet de mettre en relation des gens qui ne s'entendent pas, c'est-à-dire ne sont pas d'accord, ne se comprennent pas, voire ne se parlent et ne se lisent pas. Les polémiques permettent malgré tout une forme de mise en contact, et un mode de fonctionnement de l'espace public.

Quand on parle des fonctions de la polémique, il ne faut pas oblitérer sa visée persuasive. Si on n'essaie pas toujours de convaincre l'Opposant, c'est-à-dire celui qui représente la thèse adverse, il y a quand même une tentative de persuasion qui s'adresse au Tiers : chacun entend rallier le plus de monde possible à son point de vue. Il est vrai que la persuasion de l'adversaire réussit rarement, mais l'adresse au Tiers subsiste. Au-delà de cette fonction persuasive qui est au cœur de la rhétorique, ce que j'ai essayé de montrer, c'est que la polémique a également d'autres fonctions. Comme je l'ai déjà dit, elle tisse du lien social : elle opère les ralliements dont nous parlions tout à l'heure, elle met en contact des gens qui se retrouvent autour d'une même position à défendre, par exemple qui se rejoignent autour d'une protestation, d'un mouvement de contestation. C'est ce qu'on voit par exemple à travers les nouveaux médias. Des gens qui n'ont pas tellement l'occasion de se confronter avec d'autres ou qui, pour des raisons de sociabilité ou de convenance, n’ont pas d'occasions de débattre de façon véhémente (par exemple dans leur milieu familial ou professionnel) se retrouvent et se regroupent dans un espace virtuel où ils se confrontent à d'autres internautes. Or la confrontation - entendue comme confrontation citoyenne - est une des fonctions essentielles de la démocratie. Le fait de confronter des thèses, même de façon exacerbée, reste quelque chose d'essentiel.

On a tendance à considérer que l'argumentation et la rhétorique sont là pour rechercher de l'accord et du consensus, elles sont toujours présentées comme la possibilité de s'accorder sur le raisonnable (comme le disait Perelman). Dans la différence et devant l'impossibilité de trouver une vérité absolue, elles apparaissent comme un moyen de s'accorder sur une position qui peut apparaître comme plausible et raisonnable. Car l'accord est indispensable à la prise de décision et à l'action, sans lesquelles le processus démocratique est bloqué : le dissensus est donc le plus souvent présenté comme quelque chose de négatif. Mais j'ai essayé de montrer qu'il peut avoir des fonctions tout à fait positives; et qu'au contraire, le blocage, le gommage, le lissage du dissensus peuvent avoir des fonctions aussi bien négatives que positives : cela peut masquer un problème, empêcher la discussion, étouffer le débat contra- 
dictoire. D'ailleurs, les pays où tout le monde est toujours d'accord, et où tout le monde vote de la même manière à $99 \%$, ne sont pas les plus démocratiques...

Mots. Les langages du politique : L'autre fonction que vous évoquez dans l'ouvrage est justement de faire bouger la société, de provoquer des mouvements de contestation, de permettre le mouvement et au-delà, les évolutions?

Ruth Amossy : Je rejoins sur ce point la thèse de Chantal Mouffe (2000), qui relève d'une pensée sociologique du conflit, et avant elle, celle de Coser ${ }^{2}$. Le premier point qu'il faut relever, c'est que le conflit est quelque chose de positif, d'indispensable à la dynamique sociale. Il ne faut pas penser une société qui serait complètement dégagée de tout conflit. Simmel déjà l'avait montré mais la position de Chantal Mouffe est encore plus intéressante en ce qui me concerne car elle parle de «démocratie pluraliste agonistique», considérant que le conflit est inévitable dans la mesure où les groupes qui composent la société ont des façons de voir et des croyances différentes, voire opposées et incompatibles. Ces conflits trouvent des points d'accord transitoires, des moments d' "état de grâce», mais ensuite ils reprennent de plus belle. C'est intéressant pour nous car il faut bien gérer ces sociétés fondées sur le conflit. Et Chantal Mouffe nous dit que la meilleure façon de le faire, c'est de transformer son ennemi (quelqu'un qu'il faut combattre et anéantir) en adversaire (quelqu'un à qui on reconnaît le droit à la parole).

La différence est essentielle car un ennemi, c'est celui qu'on essaie de tuer ou d'écraser, alors qu'on laisse l'adversaire exprimer ses opinions dans un espace commun, ce qui autorise la confrontation. Et c'est là que, quand on travaille en rhétorique et en sciences du langage, on doit prendre le relais, puisque la question est de se demander comment cette transformation est gérée : la polémique est un mode de gestion du conflit dans les sociétés démocratiques, dont elle assure le bon fonctionnement et le dynamisme.

Mots. Les langages du politique : C'est le sens de ce que vous appelez dans votre ouvrage la "coexistence dans le dissensus». Pouvez-vous préciser cette notion?

Ruth Amossy: À partir du moment où on envisage la polémique comme le mode de gestion de conflits inévitables qui sont le moteur même de la démocratie, plus précisément un mode de gestion socio-discursif de ces conflits, on peut considérer bien sûr qu'elle autorise des positionnements politiques, des tentatives de persuasion, des regroupements, une dynamique de contestation et de changement social - nous avons déjà évoqué tous ces points - mais quand bien même elle ne permettrait pas tout cela, dans le pire des cas, elle autorise au moins la «coexistence dans le dissensus».

2. Voir notamment Coser, 1964 [1956] ; Simmel, 1955 [1912] (trad. fr. : 1995). 
Car il faut que des individus ou des groupes qui ont des positions fortement antagonistes puissent arriver à vivre dans le même espace - c'est ce qu'on aime aujourd'hui en France appeler le «vivre ensemble». Bien que l'argumentation ait comme objectif de résoudre les différends, il s'avère que la discussion rationnelle ne parvient pas à résoudre certains cas de désaccords profonds. II n'existe pas une seule Raison, que les hommes partagent, et à travers laquelle ils peuvent parvenir à s'entendre; i y a bien plutôt dans un même moment et dans un même espace ce que j'ai appelé des rationalités alternatives, des raisonnements fondés sur des croyances et des prémices totalement incompatibles. Cela correspond à ce que Marc Angenot appelle le «dialogue de sourds », dans l'ouvrage (2008) qui porte ce titre : des échanges qui obéissent à des logiques différentes, qui ne coïncident pas, des échanges qui ne peuvent aboutir à des points d'accord, des confrontations où chacun en vient à considérer que l'autre est un fou...

Je repars de cette idée de « dialogue de sourds » et de « ruptures cognitives ». Mais à partir de là, la question est de savoir si ces polémiques ne mènent à rien. Marc Angenot, lui, part des controverses longues, qui opposent par exemple pendant plus d'un siècle les socialistes et les anarchistes, pour montrer comment elles se poursuivent indéfiniment sans jamais déboucher sur un accord. Il en déduit que les gens continuent à polémiquer parce que chacun essaie de se positionner et de se justifier face à une sorte de... je dirais «surdestinataire», même s'il n'emploie pas ce terme, ou encore d' " auditoire universel », pour parler comme Perelman. Il remonte d'ailleurs à ce sujet aux « antilogies » antiques, et montre que l'affaire de la sophistique était de confronter ces antilogies : ce sont des positions contradictoires qui s'opposent sans qu'on puisse les départager au nom de la vérité. Ce qu'il , c'est que c'est une leçon qui peut déboucher sur un scepticisme de bon aloi : au lieu de prétendre détenir la vérité, on peut s'en tenir là et comprendre que cela fait partie d'un mode de pensée qui n'est pas de l'ordre de la recherche de la vérité absolue.

C'est en m'appuyant sur ces thèses, très séduisantes, et en les prolongeant que j'ai essayé de retrouver les fonctions sociales de la polémique. Les démocraties ont bien sûr des appareils qui permettent la prise de décision et l'action : il y a évidemment des systèmes de vote, qui varient selon les pays, des institutions représentatives comme les Parlements, qui légifèrent, et divers cadres institués dans lesquels sont prises des décisions formelles. Néanmoins, il faut aussi, pour des gens qui ne parviennent pas à s'entendre, trouver un mode de coexistence, et quand cela ne peut pas se faire dans le consensus, il faut bien que cela se fasse dans le dissensus.

J'ai pris l'exemple d'une polémique israélienne impliquant la communauté orthodoxe qui vit complètement séparée, qui a ses propres médias, n’écoute pas la télévision, etc.

Les espaces médiatiques ne sont pas partagés, c'est donc un exemple qui est un comble puisque les gens ne se parlent pas, ne se lisent pas et, littéralement, ne 
s'entendent pas. Mais en fait, il faut voir que la polémique publique n'est pas seulement un dialogue direct entre des participants qui s'affrontent. Elle se déroule dans les médias, elle est de l'ordre de la circulation des discours où de multiples voix qui ne se répondent pas dans un échange réglé se font entendre sur des plateformes différentes. Dans la mesure où elle ne se construit pas comme un dialogue direct, la polémique publique répond à une logique particulière - même dans le cas où chacun s'alimente à ses propres médias, il y a des points de rencontre, des croisements, où les gens qui refusent d'échanger peuvent au moins s'écouter et voir les positions de l'autre, fût-ce pour les combattre.

Mots. Les langages du politique : Quelles sont les conséquences de cette conception de la polémique en termes de définition de l'espace public?

Ruth Amossy : Il y a un idéal démocratique qu'il ne faut pas abandonner : je ne viens pas plaider contre le débat apaisé, rationnel, qui mène à un accord. Cela reste le but essentiel de la rhétorique, et «l'éthique de la discussion» habermassienne recherche à juste titre les modalités permettant de parvenir à un accord, d'éviter une discorde qui se traduirait en violence physique. C'est ce qu'attendait Chaïm Perelman de la rhétorique, et il ne faut pas oublier que le Traité de l'argumentation est écrit après la deuxième guerre mondiale. En aucun cas il ne faut reléguer cet idéal au grenier.

Mais à côté de cet idéal qui reste prégnant, il y a d'autres fonctionnements qui s'inscrivent dans la réalité des faits; et si on se contente de les blâmer et qu'on essaie de les exclure en les considérant comme purement négatifs, si on pense que le conflit, la gestion du conflit dans le dissensus, et la polémique doivent être éradiqués, on ne sert plus les objectifs de la démocratie pluraliste, telle que nous désirons la pratiquer, et qui repose sur la possibilité de la diversité et de la différence, donc des différends.

Il faut voir comment ce discours polémique, qui circule, construit lui aussi un espace public dans lequel les individus peuvent échanger, discuter, délibérer... Certes pas de la manière idéale qu'avait imaginée Habermas, mais, à défaut de réaliser cet idéal, d'une manière qui permet de consolider un espace démocratique, conçu peut-être différemment : un espace plus proche de ce qu'envisage Chantal Mouffe - sachant qu'ils sont eux aussi en contradiction... et qu'ils n'ont pas manqué de polémiquer!

Mots. Les langages du politique : Le discours polémique est un sujet dont vous vous êtes saisie il y a plusieurs années, avec en particulier la direction d'un certain nombre de numéros de revues. Comment les thèses que vous présentez ont-elles évolué au fil de ce parcours?

Ruth Amossy : J'ai travaillé sur la polémique parce que j'avais étudié l'argument ad hominem (dans un des premiers articles que j'ai écrits sur le sujet), avec l'idée qu'il n'est pas forcément fallacieux (c'est une idée également exprimée 
par d'autres, mais il fut un temps où les chercheurs en argumentation allaient à la chasse aux sophismes, aux fallacies). En fait l'argument ad hominem est le revers de l'ethos: l'ethos s'attache à construire de soi une image crédible tandis que l'argument ad hominem s'efforce de démolir l'image que l'autre construit de soi. Cela fait partie d'un dynamisme argumentatif qui a sa raison d'être et ne doit pas forcément être critiqué.

Deuxième étape : à un moment j'ai lancé un projet au sein de l'Académie israélienne des sciences, sur les débats sur la guerre. Et on s’était beaucoup attachés à la première guerre mondiale, en commençant par le cas classique de Romain Rolland qui s'est fait vilipender, pas seulement par la droite, mais aussi par ses amis socialistes. J'avais étudié ces arguments très violents dans une revue qui s'appelait Le Bonnet rouge, et qui disait pis que pendre de Romain Rolland. Mais ce projet envisageait aussi, de façon plus vaste, la question des débats sur la guerre...

Sur ce thème, nous avons tenu à Tel-Aviv un colloque qui a été repris dans un dossier, dirigé en collaboration avec Pierre Fiala, de la revue Mots intitulé Guerres et paix. Débats, combats, polémiques (Amossy, Fiala éd., 2004), où j'avais pu mettre l'accent sur un aspect particulier des débats sur la guerre de 14-18 : l'exacerbation des positions dans un contexte où toute entente était devenue impossible, à la différence de ce qui s'était passé au moment de la guerre de 1870. C'est un dossier auquel participaient aussi des historiens : parmi les objets abordés, l'antimilitarisme contre la religion patriotique, dont avait parlé Marc Angenot, ou encore la querelle du défaitisme, qu'avait étudiée Galit Haddad...

À partir de ce point, j'ai essayé de repenser l'argumentation dans ce sens large dont j'ai parlé, en considérant la polémique comme une modalité argumentative. Là nous avons créé une équipe de recherche, avec un nouveau projet de l'Académie israélienne des sciences (Israel Science Fondation), pour nous intéresser au fonctionnement et aux fonctions de la polémique publique. Nous avons organisé un autre colloque, avec une équipe suisse dirigée par Marcel Burger, et nous avons repris les contributions pour les publier dans un numéro de Semen qui portait sur les polémiques journalistiques (Amossy, Burger éd., 2011).

L'idée était de reprendre la réflexion en étudiant des corpus précis : chacun a travaillé sur son corpus, que ce soient les polémiques sur l'immigration, celles qui opposent partisans de la mondialisation et altermondialistes autour de la pauvreté, celle qui s'était déployée en Israël autour du désengagement de Gaza, ou d'autres polémiques médiatiques, certaines étudiées en tant qu'interactions filmées dans des débats... À ce moment-là, j'ai pu reprendre ce que j'avais fait au long des ans pour arriver à poser les jalons du livre et principalement pour expliciter cette notion de coexistence dans le dissensus, que j'avais plus particulièrement étudiée dans les forums de discussion. Ce sont toutes ces approches que j'ai essayé de rassembler dans ce livre. 
Mots. Les langages du politique : une dernière question, sur la relation entre polémique et violence. Car si on reproche aux polémiques leur inutilité (on parle de «vaines» polémiques, mais vous avez montré qu'elles n'étaient pas sivaines que cela), on les suspecte aussi de pouvoir dégénérer, en violence verbale d'abord, et même en violence physique. En ce sens elles ne seraient plus un moteur pour faire évoluer la société, mais plutôt pour l'amenervers l'affrontement, voire le chaos...

Ruth Amossy : La question reste ouverte. Tout d'abord, dans la mesure où il s'agit d'une confrontation de thèses antagonistes avec une dichotomisation, qui provoque une polarisation, la violence n'est pas impérative. J'avais beaucoup aimé, dans un ouvrage déjà ancien de 1980, un article de Nadine Gelas (1980) sur l'«hyperpolémique»: elle montrait comment dans un discours de Mitterrand, alors qu'il n'y avait pas d'attaque ouverte et même presque un lissage de la conflictualité, on avait affaire en fait à un discours très polémique. En reprenant mes corpus, j'ai voulu montrer que d'abord il est faux de dire qu'il n'y a pas d'échanges d'arguments dans les polémiques... Même si ce n'est pas de la manière que souhaiteraient les professeurs d'argumentation... Que d'autre part, le logos s'allie au pathos et que, même si on relève un engagement fort, ou des sentiments fortement ressentis, cela ne détruit pas nécessairement la rationalité du propos et ne s'accompagne pas toujours de violence verbale.

Enfin, on a parfois l'impression d'avoir affaire à des raccourcis violents qui ne reposent pas sur la Raison, alors qu'en fait le discours polémique repose sur un interdiscours nourri d'argumentaires très prégnants. Si le discours polémique ne reprend pas toujours en détail ces argumentaires, qu'il suppose connus, il ne les élimine pas et ils restent sous-jacents.

En bref la polémique est un échange, une confrontation de positions qui peut se passer de violence même si - il faut bien le dire - il est souvent accompagné de violences verbales qui peuvent apparaître à des degrés divers; et la présence de ces violences verbales en prise sur des passions ne témoigne pas nécessairement d'un manque de rationalité et d'un glissement en dehors de l'argumentation.

Mais la question de la violence verbale doit être replacée dans ses cadres: il y a des genres de discours qui ont leurs propres règles internes, relevant parfois même des rites d'interaction étudiés par les sociolinguistes. Et dans les forums de discussion par exemple, certaines pratiques sont considérées comme acceptables (dans ceux que j'ai étudiés, il y a généralement des modérateurs, car ils prennent place sur les sites de grands journaux), il y a des formes de violence verbale personnelle acceptées et acceptables, c'est-à-dire qu'il y a quand même une régulation. Ce qui est autorisé dans les forums ne le serait pas dans un cadre parlementaire, où les modalités du débat contradictoire sont fortement dissensuelles et ont leurs propres formes, elle aussi régulées, de violence (attaques de l'adversaire. discrédit jeté sur l'autre, interruptions...). 
Autre point plus problématique : quelles limites assigner à la violence? C'est évidemment un point très important, et qui a des implications graves en matière de législation : à partir de quel moment faut-il légiférer? Quels sont finalement l'apport et le bénéfice d'une législation dans ce domaine? Là, évidemment, on doit s'interroger sur les dangers de la violence verbale. Moimême, en tant qu'Israélienne, j'ai toujours à l'esprit l'exemple de l'assassinat de Yitzhak Rabin, puisqu'on avait crié à l'époque à une trop grande tolérance démocratique : dans les propos tenus contre lui, dans les manifestations qui avaient eu lieu près de son domicile et ailleurs, il y avait eu un déferlement de violence qui présentait Yitzhak Rabin avec une croix gammée ou sous les traits d'Arafat, en Palestinien ennemi d'Israël, représentations qui appelaient au meurtre. Il y a là une violence symbolique, qui est aussi celle des mouvements sociaux. À partir de quel moment risque-t-elle de se concrétiser en violence physique, ce qui retournerait alors complètement la polémique contre elle-même?

Question très difficile comme nous pouvons le voir avec l'exemple de Yitzhak Rabin : dans un numéro d'Argumentation et analyse du discours qui portait sur l'insulte (Rosier éd., 2012), il y a un article de mon collègue Eithan Orkibi, qui montre comment la contestation contre Nicolas Sarkozy, qui avait revêtu des formes violentes, devait être replacée dans une sorte de régulation qui est celle des mouvements sociaux. J'ai été frappée de voir des appels symboliques au meurtre, mais qui ont bien été perçus comme symboliques et n'ont pas été suivis d'effets. Comment fait-on la différence? Comment savons-nous à partir de quel moment il faut exclure, légalement, une forme de violence? Dans quelle mesure les choses s'autorégulent-elles, dans le sens par exemple où quelqu'un qui ferait appel à des formes de violence excessive tendrait à se discréditer lui-même? Ce sont des questions très délicates, auxquelles je n'ai pas la prétention d'apporter des réponses.

Entretien avec Ruth Amossy, professeur émérite à l'Université de Tel Aviv, 30 avril 2014. Réalisé et transcrit par Claire Oger

\section{Références}

Amossy Ruth, Fiala Pierre éd., 2004, Mots. Les langages du politique, n 76 , Guerres et paix. Débats, combats, polémiques, Lyon, ENSÉditions.

Amossy Ruth, Burger Marcel éd., 2011, Semen, n³1, Polémiques médiatiques et journalistiques. Le discours polémique en question(s).

Angenot Marc, 2008, Dialogues de sourds. Traité de rhétorique antilogique, Paris, Fayard / Mille et une nuits.

CoSer Lewis A., 1964 [1956], The Functions of Social Conflict, New York, Free Press. DASCAL Marcelo, 2008, «Dichotomies and types of debate», Controversy and confron- 
tation. Relating controversy analysis with argumentation theory, vol. VI, F. H. van Eemeren, G. Bart éd., Amsterdan, John Benjamins, p. 27-49.

GELAS Nadine, 1980, "L’hyper-polémique», Le discours polémique, N. Gelas, C. Kerbrat-Orecchioni éd., Lyon, Presses universitaires de Lyon, p.75-82.

Kerbrat-Orecchionı Catherine, 1980, "La polémique et ses définitions», N. Gelas,

C. Kerbrat-Orecchioni éd., Lyon, Presses universitaires de Lyon, Le discours polémique, p. 3-40.

Mouffe Chantal, 2000, The Democratic Paradox, Londres, New York, Verso.

Rosier Laurence éd., 2012, Argumentation et analyse du discours, revue électronique du groupe ADARR, n०8, Insulte, violence verbale, argumentation, http://aad. revues.org/1242.

Simmel Georg, 1955 [1912], Conflict, Glencoe Ill., Free Press (trad. fr. : Le conflit, DijonQuetigny, Circé, 1995). 